\title{
RNA-seq reveals more consistent reference genes for gene expression studies in human non-melanoma skin cancers
}

\author{
Van LT Hoang ${ }^{1}$ ， Lisa N Tom ${ }^{1}$ ， Xiu-Cheng Quek ${ }^{2,3}$ ， Jean-Marie Tan ${ }^{1}$ ， Elizabeth J Payne ${ }^{1}$, Lynlee L Lin ${ }^{1}$, \\ Sudipta Sinnya ${ }^{1}$, Anthony P Raphael ${ }^{1,4}{ }^{\text {, Duncan Lambie }}{ }^{5}$ ， Ian H Frazer ${ }^{6}$ ， Marcel E Dinger ${ }^{2,3}$, H. Peter \\ Soyer ${ }^{1}$, Tarl W Prow ${ }^{\text {Corresp. } 1}$ \\ 1 Dermatology Research Centre, Faculty of Medicine, Translational Research Institute, Princess Alexandra Hospital, The University of Queensland, \\ Brisbane, Queensland, Australia \\ 3 St Vincent's Clinical School, University of New South Wales, Sydney, New South Wales, Australia \\ 4 Wellman Center for Photomedicine, Harvard Medical School, Massachusetts General Hospital, Boston, Massachusetts, USA \\ 5 Department of Anatomical Pathology, Princess Alexandra Hospital, Brisbane, Queensland, Australia \\ 6 Diamantina Institute, Translational Research Institute, Princess Alexandra Hospital, The University of Queensland, Brisbane, Queensland, Australia \\ Corresponding Author: Tarl W Prow \\ Email address: t.prow@uq.edu.au
}

Identification of appropriate reference genes (RGs) is critical to accurate data interpretation in quantitative real-time PCR (qPCR) experiments. In this study, we have utilised next generation RNA sequencing (RNA-seq) to analyse the transcriptome of a panel of non-melanoma skin cancer lesions, identifying genes that are consistently expressed across all samples. Genes encoding ribosomal proteins were amongst the most stable in this dataset. Validation of this RNA-seq data was examined using qPCR to confirm the suitability of a set of highly stable genes for use as qPCR RGs. These genes will provide a valuable resource for the normalisation of qPCR data for the analysis of non-melanoma skin cancer. 
1 RNA-seq reveals more consistent reference genes for gene expression studies in human 2 non-melanoma skin cancers

3 Van L T Hoang ${ }^{1 \#}$, Lisa N Tom ${ }^{1 \#}$, Xiu-Cheng Quek ${ }^{2,3 \#}$, Jean-Marie Tan ${ }^{1}$, Elizabeth Payne ${ }^{1}$,

4 Lynlee L Lin ${ }^{1}$, Sudipta Sinnya ${ }^{1}$, Anthony P Raphael ${ }^{1,4}$, Duncan Lambie ${ }^{5}$, Ian H Frazer ${ }^{6}$, Marcel

5 E Dinger $^{2,3}$, H Peter Soyer ${ }^{1}$, Tarl W Prow ${ }^{1 *}$

7 Dermatology Research Centre, Faculty of Medicine, The University of Queensland,

8 Translational Research Institute, Princess Alexandra Hospital, Brisbane, Queensland, Australia.

$9{ }^{2}$ Garvan Institute of Medical Research, Sydney, New South Wales, Australia.

${ }^{3}$ St Vincent's Clinical School, University of New South Wales, Sydney, New South Wales,

11 Australia.

${ }^{4}$ Wellman Center for Photomedicine, Massachusetts General Hospital, Harvard Medical School, Boston, USA.

${ }^{5}$ Department of Anatomical Pathology, Princess Alexandra Hospital, Brisbane, Queensland, Australia.

17 Alexandra Hospital, Brisbane, Queensland, Australia.

\#Equal first authors

$21 *$ Corresponding Author

22 Assoc Prof Tarl Prow

23 Translational Research Institute

$24 \quad 37$ Kent Street, Level 5, Room 5082

25 Woolloongabba, QLD 4102

Email: t.prow@uq.edu.au 


\begin{abstract}
27 Abstract
28 Identification of appropriate reference genes (RGs) is critical to accurate data interpretation in 29 quantitative real-time PCR (qPCR) experiments. In this study, we have utilised next generation 30 RNA sequencing (RNA-seq) to analyse the transcriptome of a panel of non-melanoma skin 31 cancer lesions, identifying genes that are consistently expressed across all samples. Genes 32 encoding ribosomal proteins were amongst the most stable in this dataset. Validation of this 33 RNA-seq data was examined using qPCR to confirm the suitability of a set of highly stable genes 34 for use as qPCR RGs. These genes will provide a valuable resource for the normalisation of 35 qPCR data for the analysis of non-melanoma skin cancer.
\end{abstract}




\section{Introduction}

There is a growing need for identification of biomarkers of non-melanoma skin cancer (NMSC) for accurate diagnoses of skin lesions, and to predict progression and patient response to novel treatments. Quantitative real-time PCR (qPCR) is an integral technique for gene expression analysis in dermatology research, due to its high sensitivity and specificity. The expression of target genes is calculated relative to reference genes (RGs) that are stably expressed. An ideal reference should be uniformly expressed in all samples within the given experiment. Historically, selection of RGs for qPCR studies has been arbitrary, with researchers commonly selecting genes such as $18 \mathrm{~S}$ rRNA, GAPDH, and Actin without experimental validation while making the assumption that they are stably expressed across tissues. However, we now know that in many instances these commonly used RGs exhibit tissue and treatment specific variability (Chari et al. 2010; de Jonge et al. 2007). A previous preliminary study on a number of cell lines and tumour versus matched normal tissue samples showed that inappropriate choice of RGs may lead to errors when interpreting experiments involving quantitation of gene expression (Janssens et al. 2004).

Validation of RGs tailored for individual experimental conditions is therefore a necessity before commencement of gene expression studies (Bustin et al. 2009). Use of a RG whose expression is variable or changes as a result of treatment conditions invariably leads to inaccurate and misleading results. It is therefore strongly recommended in the MIQE guidelines (minimum information for publication of qPCR experiments) that suitable RGs be determined for individual experimental conditions (Bustin et al. 2009). Selecting suitable RGs is not straight forward, and as a result researchers increasingly are turning to transcriptome profiling data to identify genes that are suitable for their tissue of interest.

Analysis of gene expression patterns in skin lesions by whole transcriptome sequencing (RNAseq) is a powerful technique for the analysis of gene expression profiles (Berger et al. 2010; Jabbari et al. 2012; Wagle et al. 2014). RNA-seq allows accurate measurement of gene expression levels with a large dynamic range of expression and high signal-to-noise ratio. More importantly, RNA-seq, unlike probe-based assays (such as microarrays), provides an unbiased view of the transcriptome. As such, RNA-seq is an ideal strategy for identifying stably expressed 
67 genes suitable for use as qPCR RGs. The identification of stably expressed RGs in NMSC and 68 precancerous lesions is essential to facilitate gene expression studies.

69

We have utilised next generation transcriptome profiling by RNA-seq to identify a list of candidate genes that exhibit very low variability across a range of NMSC lesions comprising intraepidermal carcinoma (IEC), squamous cell carcinoma (SCC) and precancerous lesionsactinic keratosis (AK). The stability of these candidate genes was validated by qPCR on a panel of NMSC lesions, including AK, IEC, SCC, basal cell carcinoma (BCC) and benign seborrheic keratosis (SK). Using geNorm and Normfinder analyses, we have determined a stable combination of genes as qPCR RGs specific for skin samples. We demonstrated the importance of accurate RG selection by performing relative quantitation analysis for several targeted gene expression profiles in non-photodamaged skin, AK and SCC lesions where normalisation was performed using either new RGs together or traditional RG GAPDH.

\section{Materials and Methods}

\section{Patient samples}

Skin lesions and non-photodamaged skin tissue samples were collected from patients at the Dermatology Department at the Princess Alexandra Hospital. The study was approved by Metro South Human Research Ethics Committee and The University of Queensland Human Research Ethics Committee (HREC-11-QPAH-236, HREC-11-QPAH-477, HREC-12-QPAH-217, and HREC-12-QPAH-25). Written, informed consent was obtained from all patients prior to participation. Following biopsy, tissues were immersed in RNA later (Life Technologies, Carlsbad, CA) and stored at $-80^{\circ} \mathrm{C}$ until required. All samples were sectioned and processed according to routine protocol in the Department of Anatomical Pathology located in Princess Alexandra Hospital.

\section{RNA isolation and cDNA synthesis}

RNA isolation was performed using the Qiagen RNeasy Plus Mini kit (Qiagen GmbH, Hilden, Germany). Briefly, tissue samples were cut into small pieces, and transferred into $1.5 \mathrm{~mL}$ tube containing lysing matrix D (MP Biomedicals, Santa Ana, CA, USA) and 600uL buffer RLT containing 1\% beta-mercaptoethanol and homogenised using a Fast Prep benchtop homogeniser 
98 (MP Biomedicals, Santa Ana, CA, USA). Samples were spun 5 times using setting 6.5 for 30 99 seconds each, and chilled on ice between spins. Lysate was removed and transferred to a fresh 100 tube. For unfixed BCC samples embedded in OCT, $20 \times 10$ micron sections were cut and placed

101 into $600 \mu \mathrm{L}$ buffer RLT, and homogenised using a 18.5 gauge blunt needle attached to an

102 RNAse free syringe and resuspended 5 times. The remaining RNA extraction steps were 103 performed as above. RNA concentration was measured using the Qubit fluorometer (Life

104 Technologies, Carlsbad, CA) and RNA integrity determined using the 2100 Bioanalyser (Agilent 105 Technologies, Palo Alto, CA) on RNA Pico chips. The minimum acceptable quality for RNA for 106 analysis by qPCR was RIN >6. Complementary DNA (cDNA) was synthesised from $200 \mathrm{ng}$ total 107 RNA using the Sensifast cDNA kit (Bioline, London, UK) as per the manufacturer's instructions.

\section{Library Preparation and RNA-seq}

RNA-seq libraries of poly (A) RNA from 500ng total RNA obtained from AK, IEC and SCC and

SK samples, were generated using the TruSeq unstranded mRNA library prep KIT for Illumina multiplexed sequencing (Illumina, San Diego, CA, USA). TruSeq stranded mRNA library preparation kit was used to generate poly (A) RNA libraries for RNA obtained from normal skin samples (Illumina, San Diego, CA, USA). Libraries were sequenced (100 bp, paired-end) on the Illumina HiSeq 2500 platform and FASTQ files were analysed.

\section{Bioinformatics pipeline}

118 Sequencing data were checked for sequencing quality by FASTQC

(http://www.bioinformatics.babraham.ac.uk/projects/fastqc/). Adaptors and poor quality sequences were then removed using Trim Galore v0.3.7 ( $6 \%$ of reads removed) (http://www.bioinformatics.babraham.ac.uk/projects/trim_galore/). An average of 39.1 million trimmed reads pairs were then aligned using Tophat (V 2.1), using the unstranded protocol, against the Human Genome (hg19 build) guided with a human transcriptome generated from the GENCODE Gene annotation version 19 (Supplementary Table 1) (Harrow et al. 2012; Kim et al. 2013). Quantification of gene expression based on counting the overlaps of mapped reads with genes annotated in the GENCODE gene annotation v19 using HTSeq (Version 0.6.1p2) (Anders et al. 2015; Harrow et al. 2012) (Supplementary Table 2). Read counts were then normalised 
129 (Robinson et al. 2010; Robinson \& Oshlack 2010). Based on the Gencode gene model, an

130 expression level of 57278 genes was counted. Read counts for the remaining 57278 genes were

131 then transformed into Transcripts Per Kilobase Million (TPM) values as previously described

132 (Wagner et al. 2012) (Gene length information found in Supplementary Table 3).

133 To ensure that RGs can be detected in any of the samples, genes with any samples without

134 detectable expression level (counts $<0)$ were discarded (38670 genes discarded)

135

136 Statistical analysis for identification of RGs from RNA-seq

137 The mean TPM value of a gene is calculated by taking the average of the gene expression across

138 all samples. The CoV was measured by taking the standard deviation of expression value (TPM)

139 for a given gene divided by its mean. The ratio of the maximum to minimum (MFC) was

140 calculated using the largest TPM value divided by the smallest TPM value. A product score

141 (MFC-CoV) was calculated based on the multiplication of $\mathrm{CoV}$ and MFC value for each gene.

142 Biological Coefficient of Variance $(\mathrm{BCV})$ was obtained by calculating the square root of the tag

143 wise dispersion measured using edgeR bioconductor package(Robinson et al. 2010) normalized

144 counts per million. To facilitate exploration of the statistical analysis, data visualisation of the

145 analysis can be found on http://skinref-dev.dingerlab.org/ and the raw data on ArrayExpress

146 (https://www.ebi.ac.uk/arrayexpress/experiments/E-MTAB-5678/).

147

148

149

qPCR

150

The primers for qPCR reactions were designed using NCBI Primer BLAST

(www.ncbi.nlm.nih.gov/tools/primer-blast/) (refer to Table 1). Primers were designed to span

151

intron boundaries to avoid amplification of genomic DNA and to amplify all isoforms known to

152

each gene based on the NCBI Reference Sequence Database (RefSeq). Primers were synthesized

153 by Sigma-Aldrich (Castle Hill, Australia). qPCR reactions were performed in triplicate using 1

154 $\mu \mathrm{L}$ diluted cDNA template in a $10 \mu \mathrm{L}$ total volume. Reactions were performed in 384-well plate format on the ABI Viia7 Real-Time PCR system (Life Technologies, Carlsbad, CA, USA) using Sensifast SYBR Lo-rox mastermix (Bioline, London, UK). A 2-step cycling protocol was performed, comprising an initial 95-degree polymerase activation for 2 minutes, followed by 40 cycles of 95 degrees for 5 seconds, then 60 degrees for 20 seconds. The comparative $\mathrm{Ct}(\Delta \Delta \mathrm{Ct})$ method was used for data normalisation. 


\section{Measurement of novel RG stability}

162 The RG stability was calculated using the geNORM algorithm (Andersen et al. 2004), which is

163 integrated with qbase + software (Biogazelle, Gent, Belgium) and Normfinder software

164 (Vandesompele et al. 2002) (available as an add-in for Microsoft Word at

165 http://moma.dk/normfinder-software).

166

167

Statistical analysis of qPCR data

168

169

170

171

172

173

174

175

176

177

178

179

180

181

182

183

184

185

186

187

188

189

Statistical tests used have been described in each figure legend. Data analysis was performed using GraphPad Prism version 5.04 for Windows (GraphPad Software, Inc., La Jolla, CA, USA).

\section{Results}

\section{Identification of novel candidate RGs}

To identify RG specific for NMSC and precancerous lesions, we first performed gene expression profiling using RNA-seq on 13 AK, 7 IEC, 5 SCC lesions and 4 non-photo damaged skin samples. As previously described, a RG should show similar expression across samples, expressed at detectable levels and not display any exceptional expression in any of the samples (de Jonge et al. 2007; Eisenberg \& Levanon 2013). As with previous studies on identification of housekeeping genes, to identify genes that fall within these criteria, we measured the mean expression, CoV and the MFC for each gene within the dataset (de Jonge et al. 2007; Eisenberg $\&$ Levanon 2013) (Supplementary Table 4). The CoV measures variability within samples and the MFC is the ratio of the maximum and minimum TPM values for a given gene.

Ideally, a RG candidate should have a low $\mathrm{CoV}$ and MFC value and is expressed at detectable levels. As such, we generated a score based on the product of CoV and MFC value for each gene (MFC-CoV). We then obtained an initial list of 4643 gene candidates with a MFC-CoV that falls below the lower quartile value of MFC-CoV $\leq 1.63084$, (Supplementary Table 7). KEGG Pathway enrichment analysis on this initial gene list using geneSCF (Subhash \& Kanduri 2016) revealed that most of these stably expressed genes were involved in maintaining critical cellular activities including the citrate cycle, proteasome, RNA polymerase, protein export, RNA 
190

191

192

193

194

195

196

197

198

199

200

201

202

203

204

205

206

207

208

209

210

211

212

213

214

215

216

217

218

219

220

transport and Ribosome activities (B.H FDR Value < 1.8e-30) (Huang da et al. 2009) (Figure 1C and Supplementary Table 5-6).

To identify RGs specific for NMSC and precancerous lesions, we shortlisted 10 candidate genes from the enriched pathways involved in critical cellular activity for further validation with qPCR. In addition, we selected candidates with functions well described in literature. For instance, we chose the RPLP0 gene, whose function is not only well known for different cell and tissue types but also shown to be a suitable $\mathrm{RG}$ for research in the differentiation of human epidermal keratinocytes (Bar et al. 2009). Finally, to distinguish mRNA from genomic DNA, we selected multi-exonic genes as candidates to aid design of primers across intron boundaries.

To determine the performance of our gene candidates, we compared the $\mathrm{CoV}$, MFC and mean expression value of our $10 \mathrm{RG}$ candidates with three commonly used RGs in qPCR analysis of skin - ACTB, GAPDH and HPRT1 (Table 2) (de Kok et al. 2005). With the exception of RPL23, our candidate RGs had a lower CoV-MFC compared to the traditional RGs ACTB, HPRT1 and GAPDH (Table 2 and Figure 1A and B). RPL23 had a lower MFC but a higher CoV than GAPDH. We also calculated the BCV and found that our candidate genes fall below the mean (BCV is similar to that of the traditional house keeping genes (Supplementary Figure 1B and Supplementary Table 2).

Data visualisation of TPM, CoV and MFC metrics of the calculated genes in this study have been made available online (http://skinref-dev.dingerlab.org/) as a resource to facilitate other investigators in the community to explore the datasets and identify their candidate RG of interest (Supplementary Figure 2).

\section{qPCR validation of new RGs}

To validate and extend our findings from the RNA-seq analysis, we conducted qPCR on our 10 candidate RGs in addition to the three commonly used skin RGs ACTB, GAPDH, and HPRT1 on samples derived from a diversity of skin conditions within the spectrum of NMSC (SCC and precursors and BCC) (refer to Table 1 for list of gene primers). A total of 24 samples were tested and they include AK (4), SCC (3), SK (3), BCC (4), IEC (5), and non-photodamaged skin (5). 
221 The use of independent samples across a variety of NMSC skin lesions also minimises any

222 patient specific expression biases of our RGs. This was important as some of the lesions used in

223 RNA-seq was derived from the same patient and readings of MFC, CoV and BCV among our

224 samples could exhibit patient-specific biases. Results from the qPCR were analysed using

225 geNorm (Andersen et al. 2004) within Qbase+ software (Biogazelle) and Normfinder to

226 determine the consistency of expression values among the samples for each candidate RG

227 (Vandesompele et al. 2002).

228

229

Statistically, geNorm conduct pairwise variation (V) analysis to identify genes with the least variance between samples and is denoted as 'stability' $(M)$ values. In general, lower M values indicate lower variance in expression value among samples and genes with $\mathrm{M}$ values $\leq 0.5$ are associated with homogeneous samples. Remarkably, all of our $10 \mathrm{RG}$ candidates had $\mathrm{M}$ values $\leq$ 0.5 with RPLP0, RPL7A, RPL23, RPS27A and RPL38 ranked in the top 5 genes for geNorm M value/Stability value. In addition, to eliminate errors related to the usage of a single housekeeping gene, it is common practice to use two or more housekeeping genes. By calculating the normalization factor based on the geometric mean of multiple control genes, we identified that we need only two of our RG candidates for accurate normalization (geNorm V, $\mathrm{V} 2 / 3=0.084) . \mathrm{V}$ values of $<0.15$ indicate acceptable stability of the $\mathrm{RG}$ combination, indicating no further need for additional RGs. Amongst our RG candidates, the pair of genes with optimal normalization factor was RPL38 and RPS27A, which demonstrated the lowest M values ( 0.257 and 0.265 respectively) (Figure $2 \mathrm{~A})$.

In addition, Normfinder analysis was performed for the same dataset. Normfinder analysis performs estimation of both intra- and intergroup expression variation for each subgroup of samples (lesion types), with output given as a Stability Value. The most stable candidate was RPL7A, and the best combination of genes was RPL7A and RPLP0 (Figure 2B). Overall trends between geNorm and Normfinder analyses were similar. In both formats, the traditional RGs ACTB, GAPDH and HPRT1 were ranked as having the most variability in gene expression across the groups (increased stability values), and the genes RPLP0, RPL7A, RPL23, RPS27A and RPL38 ranked in the top 5 genes for geNorm M value/Stability value. 
252 To demonstrate the significance of our findings in NMSC research, we investigated the

253 difference in expression of keratin 17 (KRT17) in AK between normalization using our

254 candidate RGs and normalization with GAPHD (Figure 3). When using GAPDH as calibrator,

255 there was an approximate 2-fold increase in levels of KTR17 when comparing non-

256 photodamaged skin to AK (Figure 3a) or approximate 3-fold increase in SCC (Figure 3b).

257 However, this fold change was significantly higher at approximately 7-fold for AK and

258 approximately 12-fold for SCC when using the combination of RPL32 and RPS27A or either one

259 of them as RG $(P<0.05)$. There was no statistical difference between data normalised with

260 RPLP0, RPS7A or both of the two candidate genes. This result demonstrates that use of a RG,

261 which is not stably expressed, can lead to inaccurate data, particularly in instances where the

262 relative fold change is subtle.

263

264 Discussion

265 The selection of appropriate RGs is of critical importance for accurate quantification of gene

266 expression levels using qPCR. Our results concur with previous studies reporting that RNA-seq

267 is an effective method for the identification of stably expressed transcripts for applications in

268 qPCR. Through qPCR validation we demonstrate that transcriptome analysis by RNA-seq is a

269 reliable strategy for identification of genes with low variability. To the best of our knowledge,

270 this is the first study to identify suitable RGs for use in studies of pre-cancerous lesions and

271 NMSC. Our data demonstrate that the RG candidates selected for validation are stably expressed

272 in these lesions, showing strong stability in gene expression between different types of skin

273 cancer lesions and non-photo-damaged skin. Results suggest that our RNA-seq dataset is a

274 valuable resource to assemble a shortlist of candidates for validation by qPCR prior to

275 commencement of gene expression studies in NMSC and sun-damaged skin. Our RNA-seq data

276 identified many RPL and RPS genes, which encode structural proteins associated with ribosome

277 biosynthesis, as highly stable. This finding is in agreement with previous studies demonstrating

278 RPL genes as some of the least variable across a wide range of cell and tissue types. In a meta-

279 analysis of over 13,000 human gene arrays, 13 of the top 15 genes identified were ribosomal

280 structural proteins (de Jonge et al. 2007). The need for stability in this group of genes is logical

281 given that ribosome biogenesis is a tightly regulated process that is critical for fundamental

282 cellular functions including cell growth and division. 
283 We evaluated the stability of ten RGs in various NMSC samples. Results showed small

284 differences in the recommended RG combinations between geNorm and Normfinder analysis

285 outputs, but the overall stability of our candidate RGs was shown to be consistent in both

286 analyses (Figure 2). This effect is likely due to the way these algorithms are designed, each

287 utilising a different method to determine the most stable gene combinations. In the case of

288

289

290

291

292

293

294

295

296

297

298

299

300

301

302

303

304

305

306

307

308

309

310

311

312

313 geNorm, the algorithm uses pairwise correlation to determine stability, using the assumption that genes showing similar expression patterns are likely to also reflect mRNA (cDNA) levels. BestKeeper is another commonly used normalisation algorithm that is based on pairwise correlation (Pfaffl et al. 2004). A limitation of this type of normalisation process is that genes, which demonstrate co-ordinate regulation, are likely to be ranked highly, even if they are not truly stable. Normfinder is an alternative algorithm, which uses a mathematical model-based approach, which allows estimation of both intra- and intergroup expression variation to calculate a stability value. Due to this variability, it is a wise strategy to use more than one algorithm to confirm the most appropriate RGs. In our case, there is a very small variation between the highest-ranking candidates for both analytical methods. In general, any of these top ranked genes RPL38, RPL23, RPS27A, RPL7A and RPLP0 are suitable RGs for use in NMSC and precancerous lesions. By contrast, GAPDH or ACTB, which are widely used as RGs are not suitable in this type of cancer as their expression is significantly different in non-photodamaged skin and the different type of NMSC. This finding is similar to the results of a recent study that recommended not using GAPDH for normalization purposes when analysing RNA expression in human keratinocytes (Beer et al. 2015).

To observe the impact of RG stability on relative quantitation analysis, we analysed the levels of keratin KTR17 in non-photodamaged skin, SCC, and AK lesions using either GAPDH or our most stable combination as determined by Normfinder analysis, RPS7A and RPLP0. KRT17 together with KRT16 and KRT6 are involved in keratinocyte differentiation and skin cancer (Hameetman et al. 2013). It was previously reported that intermediate filament keratins in SCC lesions compared to non-photodamaged skin were upregulated (Hameetman et al. 2013; Hudson et al. 2010). In this study, we found that using different calibrators significantly altered the comparison result. The upregulation of KRT17 in AK and SCC lesions was even more pronounced with our candidate RGs. These results demonstrate that the latter set of genes can 
314 give better power in distinguishing KRT17 expression between healthy and AK or healthy and 315 SCC.

316

317 However, it should be noted that despite the high stability of our candidate RGs across a range of 318 different skin lesions, these lesions were not exposed to any treatments such as topically applied

319

320

321

322

323

324

325

326

327

328

329

330

331

332

333

334

335

336

337

338

339

340

341

342

343

344

medications, which could potentially affect their expression. A literature search should be performed prior to the commencement of the study to eliminate RGs that will potentially be affected by treatment conditions. As it is unlikely that any gene is stable across all possible experimental conditions, validation should be performed for each treatment, and in general, two or more RGs should be used to reduce the impact of any variability. For our subset of validated RGs, many are genes encoding ribosomal structural proteins. Caution should be used if considering these RGs where treatment conditions have been demonstrated to result in nucleolar stress (Nosrati et al. 2015). In this instance, selection and validation of genes with a different functional classification, such as EEF1A1 or EEF1B2, or derived from our initial long list of stable genes would be a logical strategy. Furthermore, while our RG candidates from RNA-seq has been validated to outperform traditional RGs, there were several limitations in our RNA-seq analysis. Due to the mix of stranded and unstranded RNA-seq samples used, to allow fair comparison across the samples, strand specific expression of these genes was not taken into account. Expression quantification of these RGs was conducted on a gene level, which may differ at the isoform level. Regardless, using qPCR we independently validated our RG candidates.

\section{Conclusions}

In this study, we utilized whole transcriptome RNA-seq to analyze healthy skin, precancerous and lesional NMSC for the purpose of identifying RGs, which are consistently expressed across all samples. To identify genes that fall within these criteria, we measured the mean expression, $\mathrm{CoV}$ and the MFC for each gene within the dataset. This resulted in the identification of 100 highly stable genes. To further refine the genes specific for precancerous and NMSC lesions, we then shortlisted 10 candidate genes for further validation with qPCR. These 10 candidate genes were selected based on cut-off values set lower or higher than both the mean and median values of the transcriptome. We determined that the genes RPL38, RPL23, RPS27A, RPL7A and 
345 RPLP0, which encode structural proteins associated with ribosome biosynthesis are the most suitable RGs for use in NMSC and precancerous lesions.

\section{Acknowledgements}

We would like to acknowledge the cooperation and coordination between all the members

facilities.

\section{References}

356

357

358

359

360

361

362

363

364

365

366

367

368

369

370

371

372

373

374

375

376

377

378
Anders S, Pyl PT, and Huber W. 2015. HTSeq--a Python framework to work with high-throughput sequencing data. Bioinformatics 31:166-169. 10.1093/bioinformatics/btu638

Andersen CL, Jensen JL, and Orntoft TF. 2004. Normalization of real-time quantitative reverse transcription-PCR data: a model-based variance estimation approach to identify genes suited for normalization, applied to bladder and colon cancer data sets. Cancer Res 64:5245-5250. 10.1158/0008-5472.CAN-04-0496

Beer L, Mlitz V, Gschwandtner M, Berger T, Narzt MS, Gruber F, Brunner PM, Tschachler E, and Mildner M. 2015. Bioinformatics approach for choosing the correct reference genes when studying gene expression in human keratinocytes. Experimental Dermatology 24:742-747. 10.1111/exd.12759

Berger MF, Levin JZ, Vijayendran K, Sivachenko A, Adiconis X, Maguire J, Johnson LA, Robinson J, Verhaak RG, Sougnez C, Onofrio RC, Ziaugra L, Cibulskis K, Laine E, Barretina J, Winckler W, Fisher DE, Getz G, Meyerson M, Jaffe DB, Gabriel SB, Lander ES, Dummer R, Gnirke A, Nusbaum $C$, and Garraway LA. 2010. Integrative analysis of the melanoma transcriptome. Genome Res 20:413-427. 10.1101/gr.103697.109

Bustin SA, Benes V, Garson JA, Hellemans J, Huggett J, Kubista M, Mueller R, Nolan T, Pfaffl MW, Shipley GL, Vandesompele J, and Wittwer CT. 2009. The MIQE guidelines: minimum information for publication of quantitative real-time PCR experiments. Clin Chem 55:611-622. 10.1373/clinchem.2008.112797

Chari R, Lonergan KM, Pikor LA, Coe BP, Zhu CQ, Chan TH, MacAulay CE, Tsao MS, Lam S, Ng RT, and Lam WL. 2010. A sequence-based approach to identify reference genes for gene expression analysis. BMC Med Genomics 3:32. 10.1186/1755-8794-3-32

de Jonge HJ, Fehrmann RS, de Bont ES, Hofstra RM, Gerbens F, Kamps WA, de Vries EG, van der Zee AG, te Meerman GJ, and ter Elst A. 2007. Evidence based selection of housekeeping genes. PLoS One 2:e898. 10.1371/journal.pone.0000898

de Kok JB, Roelofs RW, Giesendorf BA, Pennings JL, Waas ET, Feuth T, Swinkels DW, and Span PN. 2005. Normalization of gene expression measurements in tumor tissues: comparison of 13 endogenous control genes. Lab Invest 85:154-159. 10.1038/labinvest.3700208

Eisenberg E, and Levanon EY. 2013. Human housekeeping genes, revisited. Trends Genet 29:569-574. 10.1016/j.tig.2013.05.010 
400

401

402

403

404

405

406

407

408

409

410

411

412

413

414

415

416

417

418

419

420

421

422

423

424

425

426

427

428

429

430

Hameetman L, Commandeur S, Bavinck JNB, Wisgerhof HC, de Gruijl FR, Willemze R, Mullenders L, Tensen CP, and Vrieling H. 2013. Molecular profiling of cutaneous squamous cell carcinomas and actinic keratoses from organ transplant recipients. Bmc Cancer 13. Artn 58

10.1186/1471-2407-13-58

Harrow J, Frankish A, Gonzalez JM, Tapanari E, Diekhans M, Kokocinski F, Aken BL, Barrell D, Zadissa A, Searle S, Barnes I, Bignell A, Boychenko V, Hunt T, Kay M, Mukherjee G, Rajan J, Despacio-Reyes G, Saunders G, Steward C, Harte R, Lin M, Howald C, Tanzer A, Derrien T, Chrast J, Walters N, Balasubramanian S, Pei B, Tress M, Rodriguez JM, Ezkurdia I, van Baren J, Brent M, Haussler D, Kellis M, Valencia A, Reymond A, Gerstein M, Guigo R, and Hubbard TJ. 2012. GENCODE: the reference human genome annotation for The ENCODE Project. Genome Res 22:1760-1774. 10.1101/gr.135350.111

Huang da W, Sherman BT, and Lempicki RA. 2009. Bioinformatics enrichment tools: paths toward the comprehensive functional analysis of large gene lists. Nucleic Acids Res 37:1-13. 10.1093/nar/gkn923

Hudson LG, Gale JM, Padilla RS, Pickett G, Alexander BE, Wang J, and Kusewitt DF. 2010. Microarray Analysis of Cutaneous Squamous Cell Carcinomas Reveals Enhanced Expression of Epidermal Differentiation Complex Genes. Molecular Carcinogenesis 49:619-629. 10.1002/mc.20636

Jabbari A, Suarez-Farinas M, Dewell S, and Krueger JG. 2012. Transcriptional profiling of psoriasis using RNA-seq reveals previously unidentified differentially expressed genes. J Invest Dermatol 132:246-249. 10.1038/jid.2011.267

Janssens N, Janicot M, Perera T, and Bakker A. 2004. Housekeeping genes as internal standards in cancer research. Mol Diagn 8:107-113.

Kim D, Pertea G, Trapnell C, Pimentel H, Kelley R, and Salzberg SL. 2013. TopHat2: accurate alignment of transcriptomes in the presence of insertions, deletions and gene fusions. Genome Biol 14:R36. 10.1186/gb-2013-14-4-r36

Nosrati N, Kapoor NR, and Kumar V. 2015. DNA damage stress induces the expression of Ribosomal Protein S27a gene in a p53-dependent manner. Gene 559:44-51. 10.1016/j.gene.2015.01.014

Pfaffl MW, Tichopad A, Prgomet C, and Neuvians TP. 2004. Determination of stable housekeeping genes, differentially regulated target genes and sample integrity: BestKeeper--Excel-based tool using pair-wise correlations. Biotechnol Lett 26:509-515.

Robinson MD, McCarthy DJ, and Smyth GK. 2010. edgeR: a Bioconductor package for differential expression analysis of digital gene expression data. Bioinformatics 26:139-140. 10.1093/bioinformatics/btp616

Robinson MD, and Oshlack A. 2010. A scaling normalization method for differential expression analysis of RNA-seq data. Genome Biol 11:R25. 10.1186/gb-2010-11-3-r25

Subhash S, and Kanduri C. 2016. GeneSCF: a real-time based functional enrichment tool with support for multiple organisms. BMC Bioinformatics 17. 10.1186/s12859-016-1250-z

Vandesompele J, De Preter K, Pattyn F, Poppe B, Van Roy N, De Paepe A, and Speleman F. 2002. Accurate normalization of real-time quantitative RT-PCR data by geometric averaging of multiple internal control genes. Genome Biol 3:RESEARCH0034.

Wagle N, Van Allen EM, Treacy DJ, Frederick DT, Cooper ZA, Taylor-Weiner A, Rosenberg M, Goetz EM, Sullivan RJ, Farlow DN, Friedrich DC, Anderka K, Perrin D, Johannessen CM, McKenna A, Cibulskis K, Kryukov G, Hodis E, Lawrence DP, Fisher S, Getz G, Gabriel SB, Carter SL, Flaherty KT, Wargo JA, and Garraway LA. 2014. MAP kinase pathway alterations in BRAF-mutant melanoma patients with acquired resistance to combined RAF/MEK inhibition. Cancer Discov 4:61-68. 10.1158/2159-8290.CD-13-0631 
431 Wagner GP, Kin K, and Lynch VJ. 2012. Measurement of mRNA abundance using RNA-seq data: RPKM 432

433 measure is inconsistent among samples. Theory in Biosciences 131:281-285. 10.1007/s12064012-0162-3

434 
Table $\mathbf{1}$ (on next page)

Reference gene qPCR primers designed using NCBI Primer BLAST 


\begin{tabular}{|c|c|c|c|c|}
\hline Gene & $\begin{array}{l}\text { Accession } \\
\text { number }\end{array}$ & Forward primer & Reverse primer & $\begin{array}{l}\text { Amplic } \\
\text { on size } \\
\text { (bp) }\end{array}$ \\
\hline RPL9 & $\begin{array}{l}\text { NM_00066 } \\
1.4\end{array}$ & $\begin{array}{l}\text { CTGCGTCTACTGCGAGAA } \\
\text { TGA }\end{array}$ & $\begin{array}{l}\text { CACGATAACTGTGCGTC } \\
\text { CCT }\end{array}$ & 98 \\
\hline $\begin{array}{l}\text { RPL3 } \\
8\end{array}$ & $\begin{array}{l}\text { NM_00099 } \\
9.3\end{array}$ & $\begin{array}{l}\text { GCCATGCCTCGGAAAATT } \\
\text { G }\end{array}$ & $\begin{array}{l}\text { CCAGGGTGTAAAGGTAT } \\
\text { CTGC }\end{array}$ & 139 \\
\hline $\begin{array}{l}\text { RPL1 } \\
1\end{array}$ & $\begin{array}{l}\text { NM_00097 } \\
5.3\end{array}$ & $\begin{array}{l}\text { AGAAGGGTCTAAAGGTG } \\
\text { CGG }\end{array}$ & $\begin{array}{l}\text { AGTCCAGGCCGTAGATA } \\
\text { CCA }\end{array}$ & 138 \\
\hline $\begin{array}{l}\text { RPL2 } \\
3\end{array}$ & $\begin{array}{l}\text { NM_00097 } \\
8.3\end{array}$ & $\begin{array}{l}\text { TCCAGCAGTGGTCATTCG } \\
\text { AC }\end{array}$ & $\begin{array}{l}\text { GCAGAACCTTTCATCTC } \\
\text { GCC }\end{array}$ & 117 \\
\hline $\begin{array}{l}\text { EEF1 } \\
\text { B2 }\end{array}$ & $\begin{array}{l}\text { NM_00195 } \\
9.3\end{array}$ & $\begin{array}{l}\text { AGTATTTGAAGCCGTGTC } \\
\text { CAG }\end{array}$ & $\begin{array}{l}\text { ACATCGGCAGGACCATA } \\
\text { TTTG }\end{array}$ & 144 \\
\hline $\begin{array}{l}\text { RPS27 } \\
\text { A }\end{array}$ & $\begin{array}{l}\text { NM_00295 } \\
4.5\end{array}$ & $\begin{array}{l}\text { ACCACTCCCAAGAAGAA } \\
\text { TAAGC }\end{array}$ & $\begin{array}{l}\text { ACTTGCCATAAACACCC } \\
\text { CAG }\end{array}$ & 147 \\
\hline $\begin{array}{l}\text { RPL7 } \\
\text { A }\end{array}$ & $\begin{array}{l}\text { NM_00097 } \\
2.2\end{array}$ & $\begin{array}{l}\text { GGCATTGGACAGGACAT } \\
\text { CCA }\end{array}$ & $\begin{array}{l}\text { AGGCACTTTCAGCCGCT } \\
\text { TAT }\end{array}$ & 114 \\
\hline RPS 13 & $\begin{array}{l}\text { NM_00101 } \\
7.2\end{array}$ & $\begin{array}{l}\text { TCCCCACTTGGTTGAAGT } \\
\text { TGA }\end{array}$ & $\begin{array}{l}\text { AGGAGTAAGGCCCTTCT } \\
\text { TGG }\end{array}$ & 77 \\
\hline $\begin{array}{l}\text { EEF1 } \\
\text { A1 }\end{array}$ & $\begin{array}{l}\text { NM_00140 } \\
2.5\end{array}$ & $\begin{array}{l}\text { GAAAGCTGAGCGTGAAC } \\
\text { GTG }\end{array}$ & $\begin{array}{l}\text { AGTCAGCCTGAGATGTC } \\
\text { CCT }\end{array}$ & 143 \\
\hline $\begin{array}{l}\text { RPLP } \\
0\end{array}$ & $\begin{array}{l}\text { NM_00100 } \\
2.3\end{array}$ & $\begin{array}{l}\text { ATCAACGGGTACAAACG } \\
\text { AGTC }\end{array}$ & $\begin{array}{l}\text { CAGATGGATCAGCCAAG } \\
\text { AAGG }\end{array}$ & 97 \\
\hline $\begin{array}{l}\text { GAPD } \\
\mathrm{H}\end{array}$ & $\begin{array}{l}\text { NM_00204 } \\
6.5\end{array}$ & $\begin{array}{l}\text { CCCACTCCTCCACCTTTG } \\
\text { AC }\end{array}$ & $\begin{array}{l}\text { TTCCTCTTGTGCTCTTGC } \\
\text { TG }\end{array}$ & 180 \\
\hline $\begin{array}{l}\text { HPRT } \\
1\end{array}$ & $\begin{array}{l}\text { NM_00019 } \\
4.2\end{array}$ & $\begin{array}{l}\text { TGCTGAGGATTTGGAAA } \\
\text { GGG }\end{array}$ & $\begin{array}{l}\text { ACAGAGGGCTACAATGT } \\
\text { GATG }\end{array}$ & 115 \\
\hline ACTB & $\begin{array}{l}\text { NM_00110 } \\
1.3\end{array}$ & $\begin{array}{l}\text { ACCTTCTACAATGAGCTG } \\
\text { CG }\end{array}$ & $\begin{array}{l}\text { CCTGGATAGCAACGTAC } \\
\text { ATGG }\end{array}$ & 148 \\
\hline
\end{tabular}

2 


\section{Table 2 (on next page)}

RNA-seq scoring of selected candidate reference genes and commonly used reference genes.

RNA-seq scoring of selected candidate reference genes and commonly used reference genes, ranked on CoV (coefficient of variation) score, mean $=$ mean expression value, $\mathrm{MFC}=$ maximum fold change calculated using transcript per million values. Candidates are ranked from the smallest to largest CoV values. 
1

\begin{tabular}{|l|l|l|l|}
\hline $\begin{array}{l}\text { Gene } \\
\text { Symbol }\end{array}$ & CoV & Mean & MFC \\
\hline RPS13 & 0.25929018 & $\mathbf{4 7 1 . 6 3 2 8}$ & $\mathbf{2 . 9 4 5 3}$ \\
\hline RPL7A & 0.270464492 & $\mathbf{1 0 5 2 . 6 6 1 3}$ & $\mathbf{3 . 0 5 8 9}$ \\
\hline EEF1B2 & 0.289246663 & $\mathbf{5 6 9 . 7 8 2 9}$ & $\mathbf{2 . 9 7 0 3}$ \\
\hline RPS27A & 0.292810337 & $\mathbf{6 2 5 . 2 5 7 1}$ & $\mathbf{3 . 2 9 1 5}$ \\
\hline RPLP0 & 0.300470977 & $\mathbf{1 6 6 . 6 7 5 8}$ & $\mathbf{3 . 0 2 1 4}$ \\
\hline RPL38 & 0.307936407 & $\mathbf{1 3 3 6 . 0 4 0 7}$ & $\mathbf{2 . 7 4 0 1}$ \\
\hline EEF1A1 & 0.312400695 & $\mathbf{2 8 0 9 . 6 3 0 6}$ & $\mathbf{3 . 5 4 6 9}$ \\
\hline RPL11 & 0.325188306 & $\mathbf{9 2 6 . 3 6 7 8}$ & $\mathbf{3 . 8 0 9 0}$ \\
\hline RPL9 & 0.336714133 & $\mathbf{4 8 9 . 1 8 9 6}$ & $\mathbf{3 . 9 2 2 0}$ \\
\hline GAPDH & 0.352954726 & $\mathbf{8 6 5 . 5 2 7 8}$ & $\mathbf{4 . 2 3 4 4}$ \\
\hline RPL23 & 0.372401947 & $\mathbf{2 4 1 . 0 3 7 2}$ & $\mathbf{3 . 9 1 2 4}$ \\
\hline HPRT1 & $\mathbf{0 . 3 9 6 0}$ & $\mathbf{3 5 . 4 3 9 6}$ & $\mathbf{1 1 . 0 4 2 3}$ \\
\hline ACTB & $\mathbf{0 . 4 2 6 0}$ & $\mathbf{2 0 0 5 . 6 0 1 2}$ & $\mathbf{1 0 . 9 5 8 8}$ \\
\hline
\end{tabular}

2 


\section{Figure $\mathbf{1}$ (on next page)}

RNA-seq analysis of genes and candidate reference genes
A) Scatterplot comparing Coefficient of variation (CoV) values against mean expression values (log2) transformed and represented in Transcript per kilobases million (TPM) for genes detected using the RNA-seq of patient derived skin samples. Each gene is represented by a single dot. Genes selected for validation to function as reference genes in non-melanoma skin cancers (NMSC) and precancerous lesions are shown in Black. Reference genes commonly used in the literature, ACTB (Red), GAPDH (Blue) and HPRT1 (Green), are also highlighted for comparisons. B) Comparison of maximum fold change score in gene expression of candidate reference genes and traditional reference genes. C) Results of KEGG pathway enrichment analysis conducted with a list of 3714 genes found with product score between each gene's MFC and CoV score below the lower quantile. Enrichment percentage is defined as the percentage of genes in the pathway that are overlaps with genes in our list. D) Boxplot showing expression value from RNASeq experiment of 29 skin lesions of selected reference genes candidate (blue) with commonly used housekeeping genes ACTB, GAPHD and HPRT1 (red). 
A)

Comparison Between CoV against Pecer $\log _{2}$ TPM of genes expression

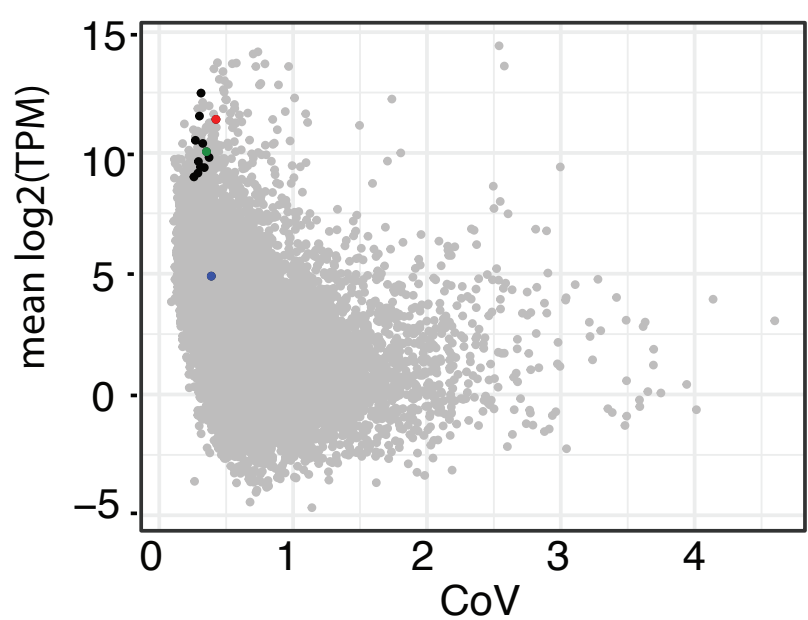

OGAPDH OACTB OHPRT1

Validated Genes
B)

MFC Differences Between Validated and Traditiona! ReferenceGenes

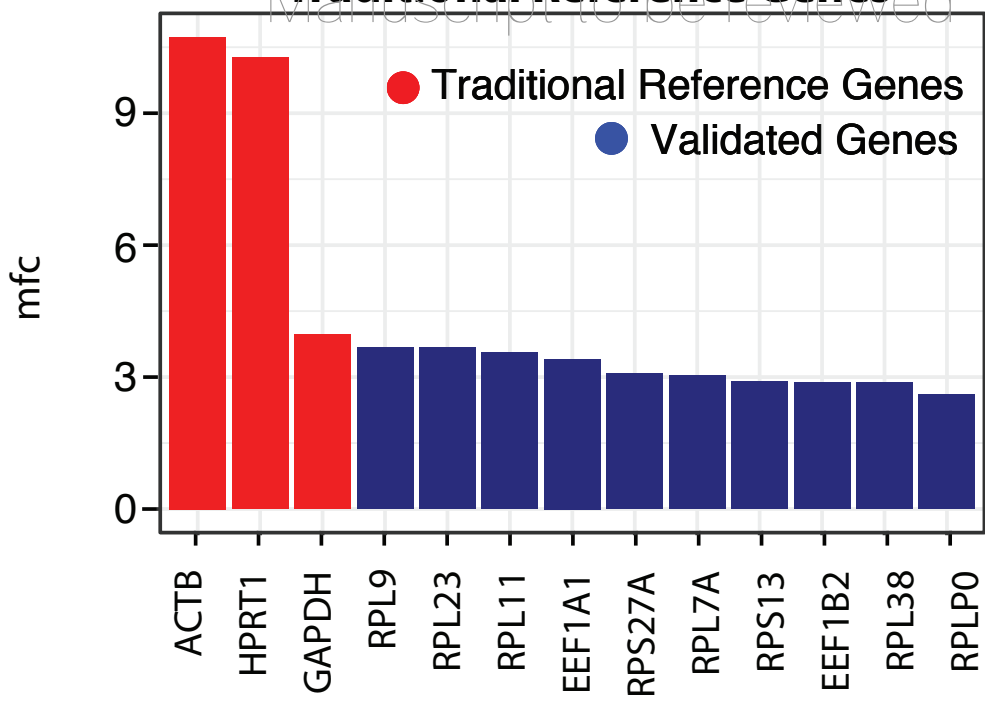

C)

\section{Enriched KEGG Pathway ( FDR > 0.05 )}

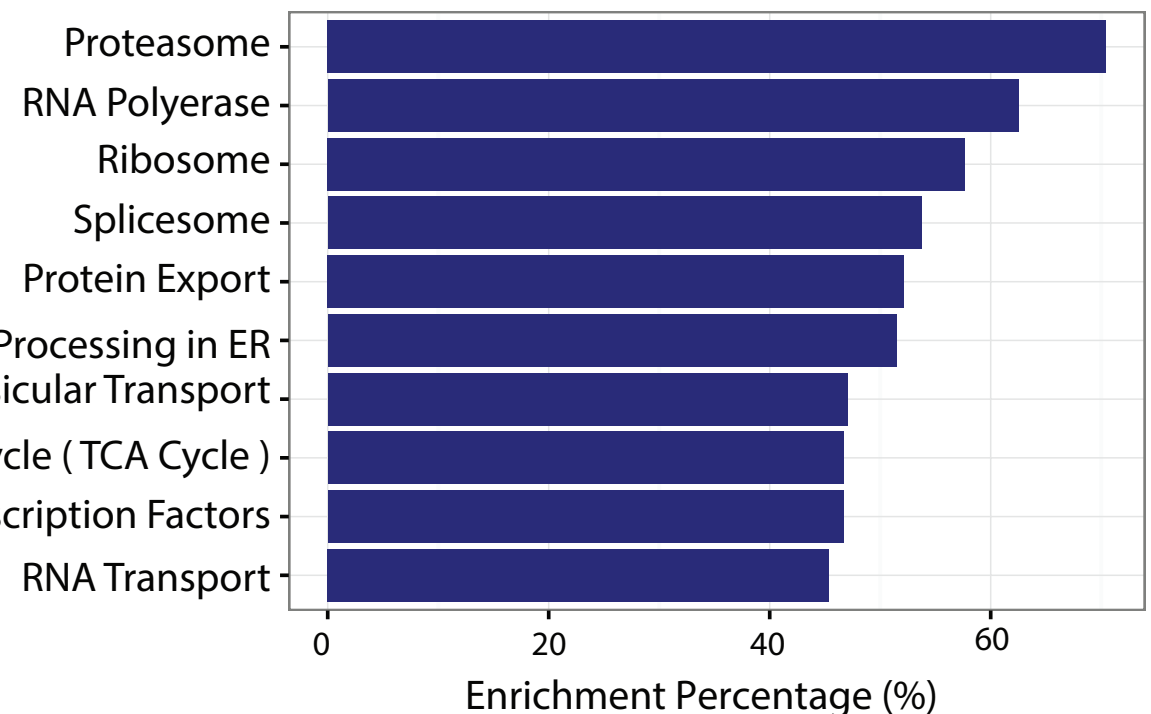

D)

\section{Expression Values Of Reference Genes Candidates}

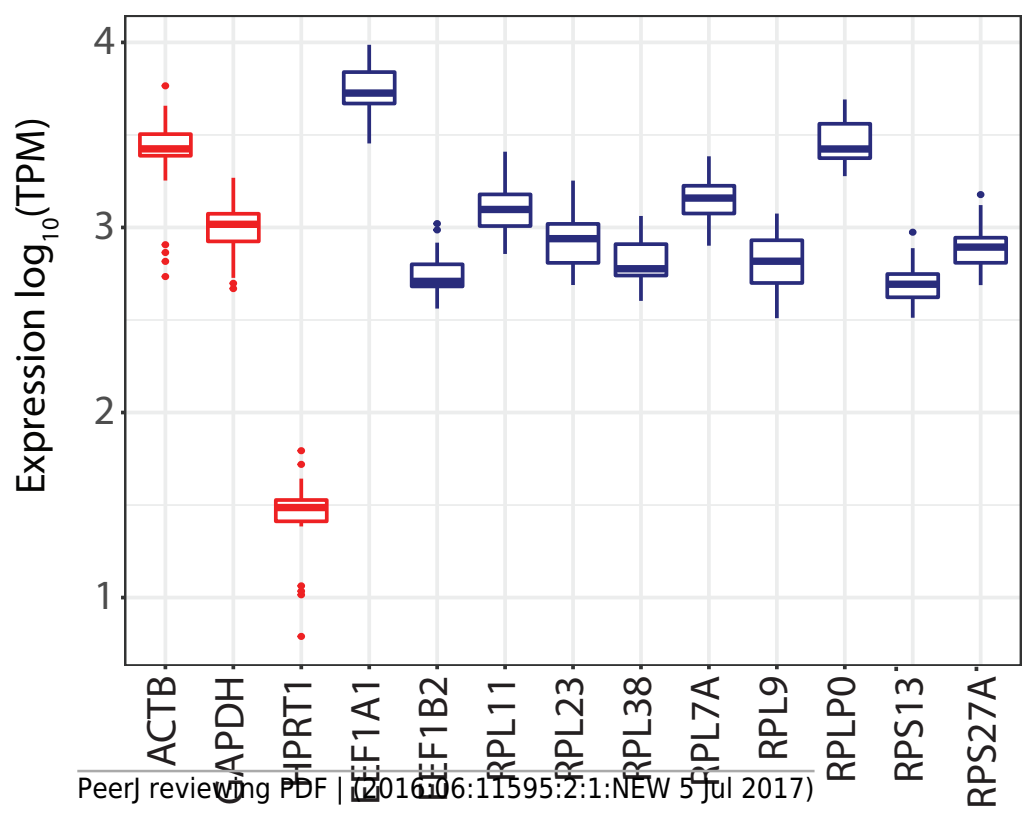

Validated Genes

Traditional Reference Genes 


\section{Figure 2 (on next page)}

Comparison of expression stability using GeNorm and Normfinder
A) Average expression stability of reference targets (geNorm). geNorm M value, an indicator of gene expression stability, was determined using the geNorm algorithm. Decreasing values correlate with smaller variations in gene expression levels across lesion groups AK, SCC, SK, BCC, IEC, and healthy skin. B) Average expression stability of reference targets (Normfinder). Stability values were determined for each gene using the Normfinder algorithm. Decreasing values correlate with smaller variations in gene expression levels across lesion groups AK, SCC, SK, BCC, IEC, and healthy skin. 

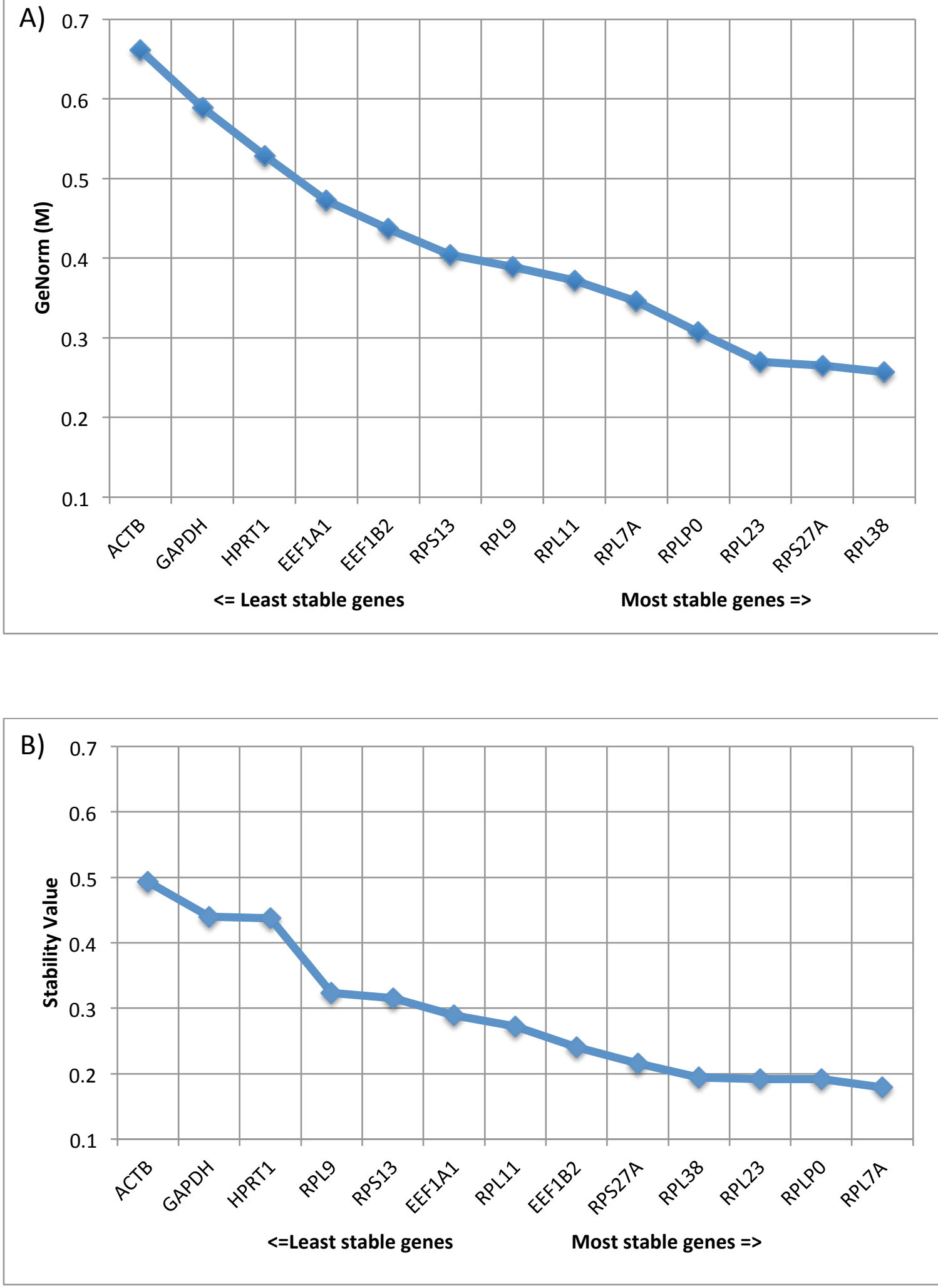
Figure 3

KRT17 levels in precancerous and lesional NMSC

Comparison of relative quantitation analysis of KRT17 levels in AK (a) and SCC (b) lesions using either RPS7A/RPLP0 or GAPDH as the reference gene relative to non-photodamaged skin. Data are presented as mean $\pm \mathrm{SEM}, \mathrm{n}=3, *$ indicates $P<0.05$; one-way ANOVA and Turkey post-test.

A)

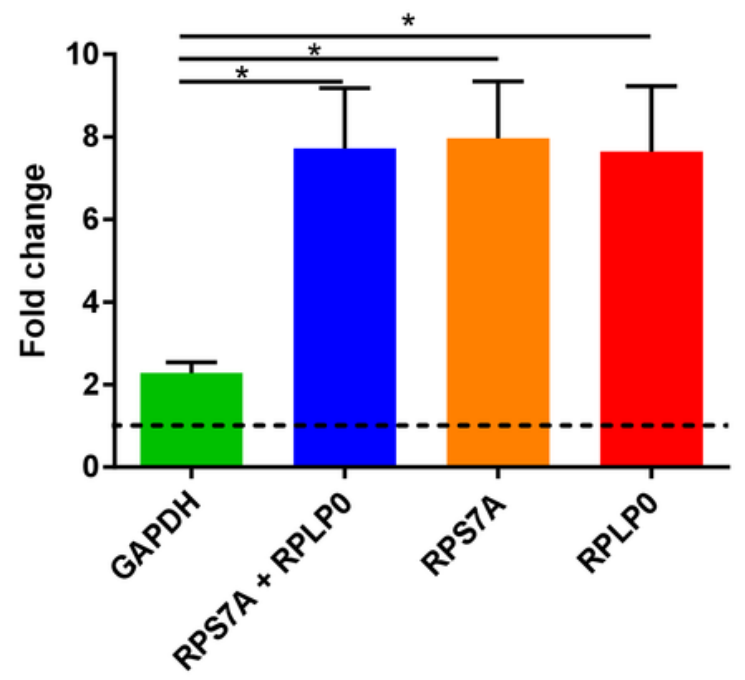

B)

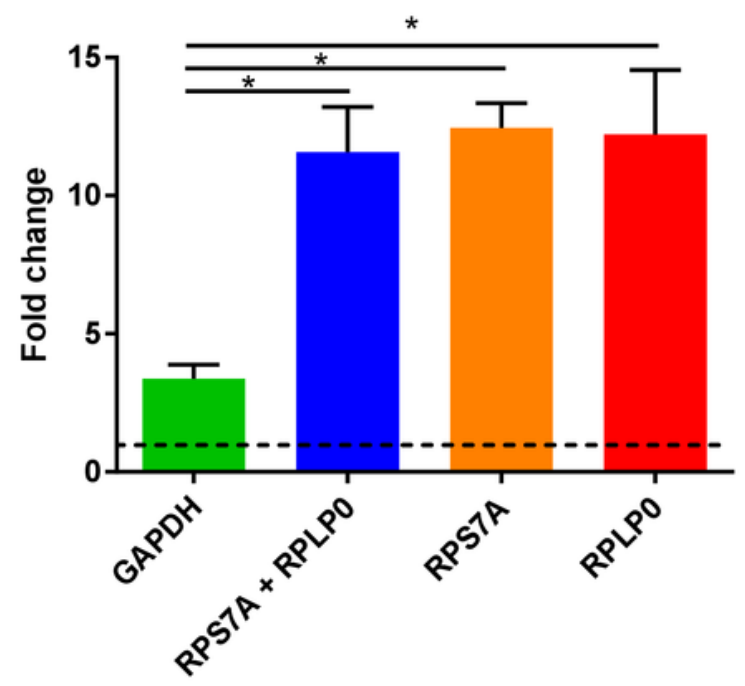

